\title{
Determination of Soil Moisture Beneath a Stalk or Trunk Dominated Canopy
}

K. C. McDonald, M. C. Dobson and F. T. Ulaby

Radiation Laboratory

Department of Electrical Engineering and Computer Science The University of Michigan

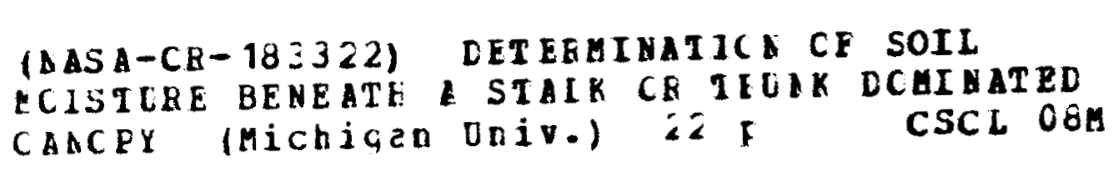

N89-14621

$63 / 46 \quad \begin{array}{ll}\text { Unclas } \\ 0169983\end{array}$ 


\section{OVERVIEW}

1. Radiative transfer model

- Simplified L-Band MIMICS model

- $\sigma_{H H}^{0}, \sigma_{V V}^{0}$ and $\Delta \phi_{H H-V V}$

2. Example model computations

- Dominant backscatter contributions

3. Application of the model to determining soil moisture

- Inversion of backscatter data 
ORIONAR PAGE IS

OF POOR QUALITY

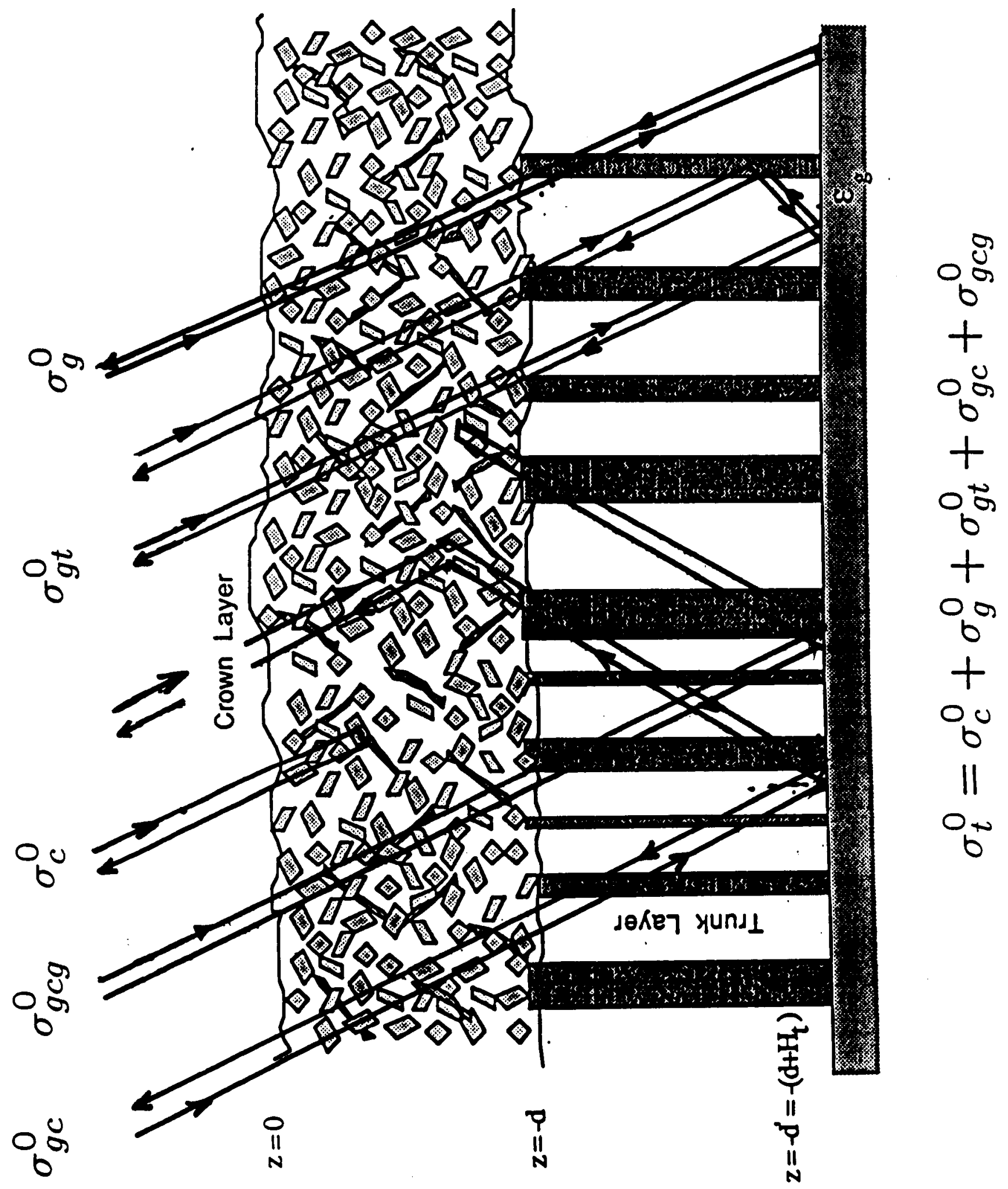


- Direct Crown Backscatter

$$
\begin{gathered}
\sigma_{c}^{0}=\frac{\sigma_{v} \cos \theta}{2 \kappa_{e}}\left[1-\exp \left(-\frac{2 \kappa_{e} H_{c}}{\cos \theta}\right)\right] \\
\sigma_{v}=\frac{3}{2} \omega \simeq \frac{3}{2}(0.1) \\
\kappa_{e}^{p}=\kappa_{l}+\kappa_{t}^{p} \\
\kappa_{l}=\operatorname{Im}\left[\frac{4 \pi}{\lambda} \sqrt{1+\frac{v_{l}}{3}\left(\epsilon_{l}-1\right)\left(2+\frac{1}{\epsilon_{l}}\right)}\right] \\
\kappa_{t}^{p}=-N \frac{2 \lambda}{\pi} \operatorname{Re}\left[\sum_{n=-\infty}^{\infty} C_{n}^{p}\left(\theta_{0}\right)\right] \\
p=v \text { or } h
\end{gathered}
$$




\section{- Direct Ground Backscatter}

\section{- Small Perturbation Model}

$$
\begin{aligned}
\sigma_{g}^{0} & =\sigma_{s, p}^{0} \exp \left(-2 \kappa_{e}^{p} \sec \theta\right) \\
\sigma_{s, p}^{0} & =4(k s)^{2}(k l)^{2} \cos ^{4} \theta\left|\alpha_{p p}\right|^{2} e^{-(k l \sin \theta)^{2}}
\end{aligned}
$$

$$
\alpha_{h h}=\frac{\cos \theta-\sqrt{\epsilon_{s}-\sin ^{2} \theta}}{\cos \theta+\sqrt{\epsilon_{s}-\sin ^{2} \theta}}
$$

$$
\alpha_{v v}=\left(\epsilon_{s}-1\right) \frac{\sin ^{2} \theta-\epsilon_{s}\left(1+\sin ^{2} \theta\right)}{\left[\epsilon_{s} \cos \theta+\sqrt{\epsilon_{s}-\sin ^{2} \theta}\right]^{2}}
$$

Validity Conditions Recommended Conditions

$$
\begin{array}{rlrl}
s & \leq 0.05 \lambda & l \leq 0.25 \lambda \\
m & =\sqrt{2} \frac{s}{l} \leq 0.3 & & s \leq 0.05 \lambda \\
l & \leq 0.5 \lambda & &
\end{array}
$$


- Ground-Trunk (Stalk) Interaction

$$
\begin{gathered}
\sigma_{g t, p p}^{0}=4 N e^{-2 \kappa_{e}^{p} H_{c} \sec \theta}\left|R_{p}(\theta)\right|^{2} \sigma_{p}^{t} \\
\sigma_{p}^{t}(\theta)=\frac{16 H_{t}^{2}}{\pi}\left|\sum_{n=-\infty}^{\infty}(-1)^{n} C_{n}^{p}\right|^{2} \\
p=v \text { or } h
\end{gathered}
$$

Validity Conditions:

$-H_{t} \gg \lambda$

- Specular forward scatter from soil 
Backscatter Contributions, $\theta=50$ Degrees
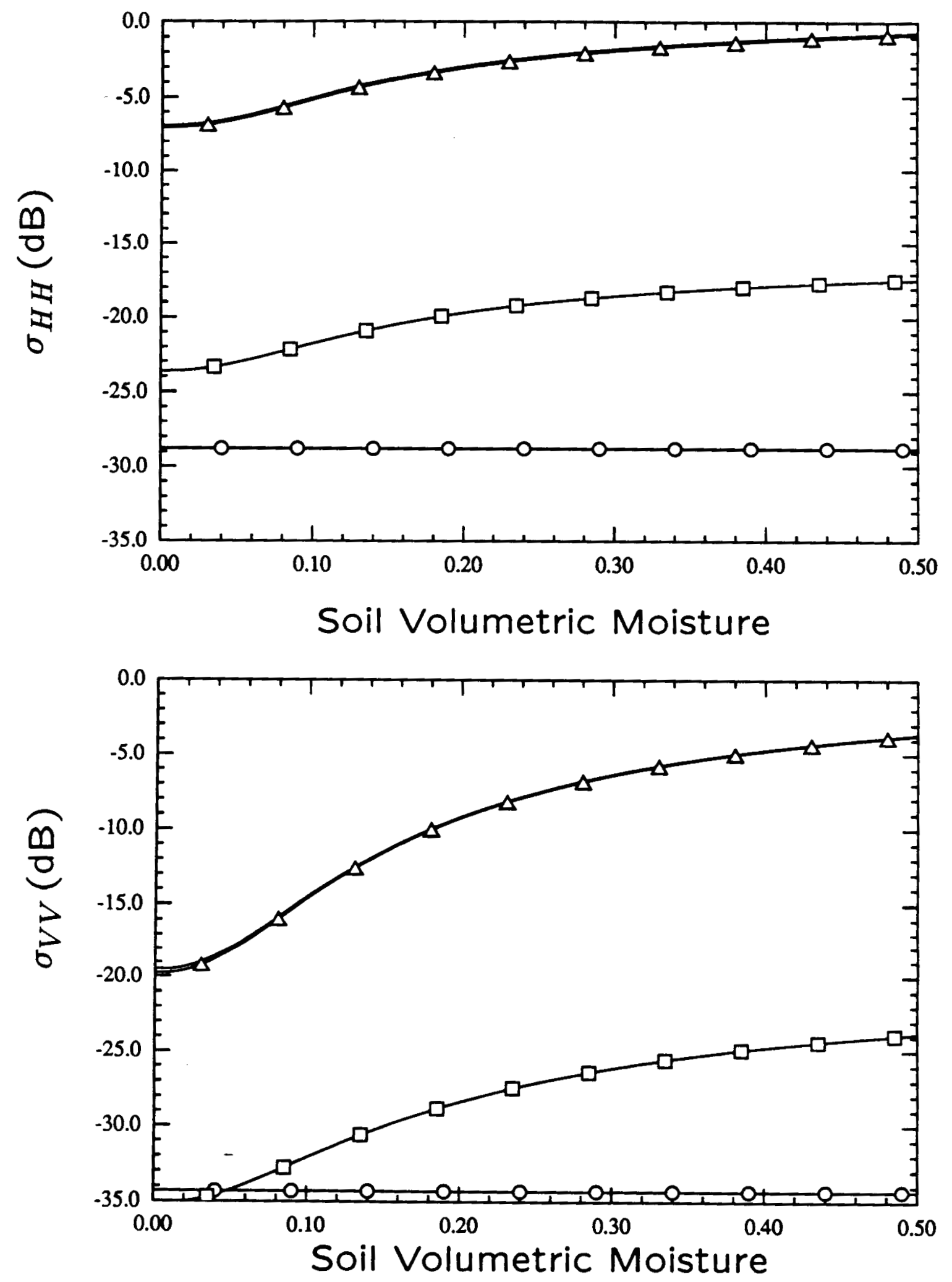

- Total Backscatter

ODirect Crown

$\square$ Direct Ground

Stalk Moisture $=0.5$

$\triangle$ Ground-Stalk 


\section{Backscatter Contributions, $\theta=30$ Degrees}
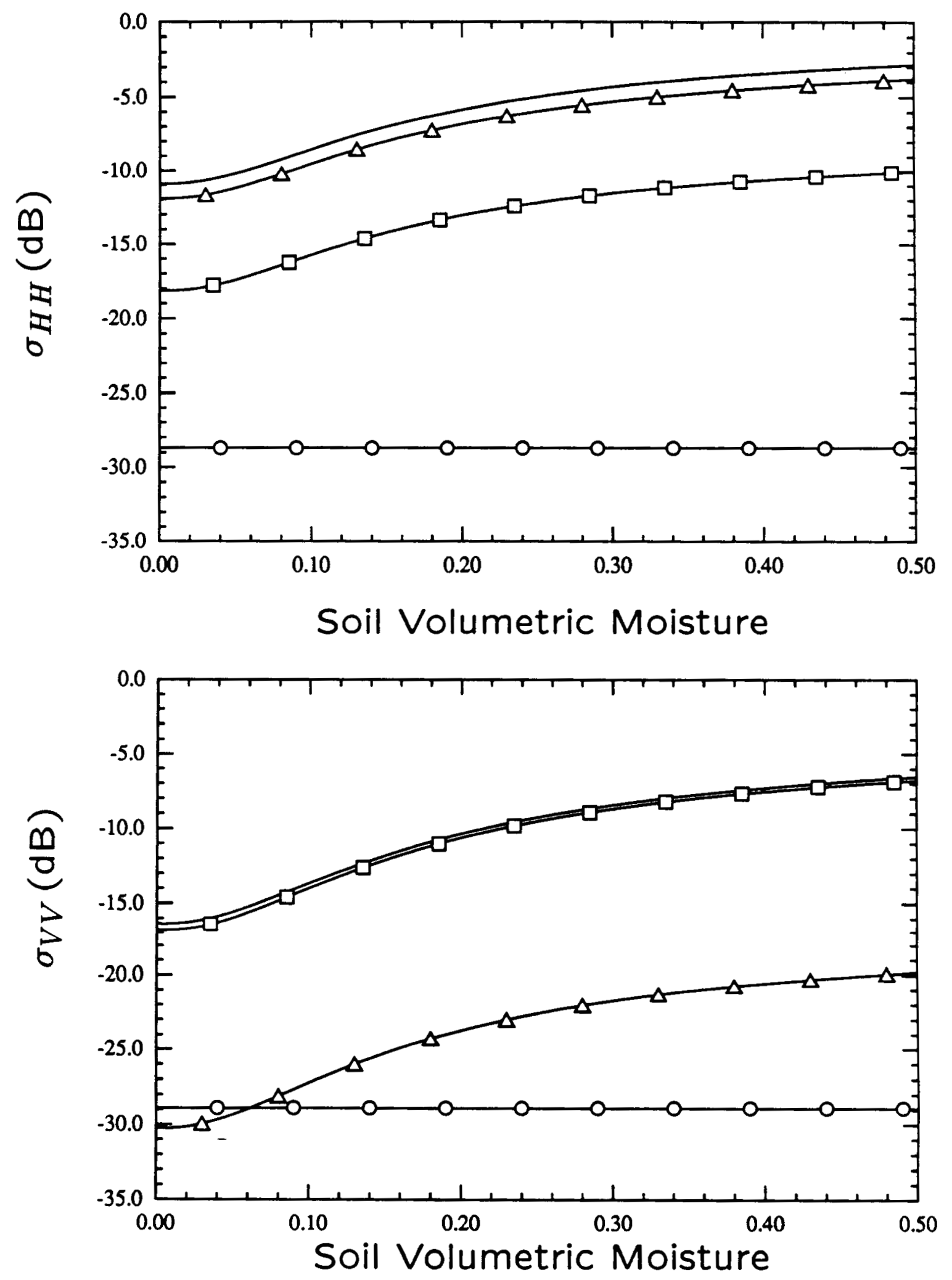

- Total Backscatter

ODirect Crown

$\square$ Direct Ground

Stalk Moisture $=0.2$

$\triangle$ Ground-Stalk 


\section{Polarization Phase Difference}

$$
\begin{aligned}
\Delta \phi_{(H H-V V) g s} & =2 \Delta \phi_{p}+\Delta \phi_{g, s}+\Delta \phi_{t r} \\
\Delta \phi_{(H H-V V) g} & =2 \Delta \phi_{p}+\Delta \phi_{g, b}
\end{aligned}
$$

- Propagation Phase Difference

$$
\begin{gathered}
\Delta \phi_{p}=\frac{2 N H_{t}}{k} \tan \theta\left[\operatorname{Im}\left\{A_{h}(\theta, \pi)\right\}-\operatorname{Im}\left\{A_{v}(\theta, \pi)\right\}\right] \\
A_{p}(\theta, \pi)=\sum_{n=-\infty}^{\infty} C_{n}^{p}(\theta), \quad p=v \text { or } h
\end{gathered}
$$

- Trunk Scatter Phase Difference

$$
\begin{array}{r}
\Delta \phi_{t}=\tan ^{-1}\left\{\frac{\operatorname{Im}\left[A_{h} / A_{v}\right]}{\operatorname{Re}\left[A_{h} / A_{v}\right]}\right\} \\
A_{p}\left(\theta, 0^{\circ}\right)=\sum_{n=-\infty}^{\infty}(-1)^{n} C_{n}^{p}(\theta) \\
p=v \text { or } h
\end{array}
$$


- Ground Scatter Phase Difference

- Specular Scatter

$$
\Delta \phi_{g, s}=\tan ^{-1}\left\{\frac{\operatorname{Im}\left(R_{h} / R_{v}\right)}{\operatorname{Re}\left(R_{h} / R_{v}\right)}\right\}-180^{\circ}
$$

- Backscatter

$$
\Delta \phi_{g, b}=\tan ^{-1}\left\{\frac{\operatorname{Im}\left(\alpha_{h h} / \alpha_{v v}\right)}{\operatorname{Re}\left(\alpha_{h h} / \alpha_{v v}\right)}\right\}
$$




\section{Total Phase Difference}

$\left[\begin{array}{cccc}\left|S_{V V}\right|^{2} & 0 & 0 & 0 \\ 0 & \left|S_{H H}\right|^{2} & 0 & 0 \\ 0 & 0 & \operatorname{Re}\left(S_{V V} S_{H H}^{*}\right) & -\operatorname{Im}\left(S_{V V} S_{H H}^{*}\right) \\ 0 & 0 & \operatorname{Im}\left(S_{V V} S_{H H}^{*}\right) & \operatorname{Re}\left(S_{V V} S_{H H}^{*}\right)\end{array}\right]$

where:

$$
\begin{aligned}
\left|S_{V V}\right|^{2} & =\sigma_{g t, V V}^{0}+\sigma_{g, V V}^{0} \\
\left|S_{H H}\right|^{2} & =\sigma_{g t, H H}^{0}+\sigma_{g, H H}^{0}
\end{aligned}
$$

$\operatorname{Re}\left(S_{V V} S_{H H}^{*}\right)=\operatorname{Re}\left[\sqrt{\sigma_{g t, V V}} \sqrt{\sigma_{g t, H H}} e^{i \Delta \phi_{g s}}\right]$

$+\operatorname{Re}\left[\sqrt{\sigma_{g, V V}} \sqrt{\sigma_{g, H H}} e^{i \Delta \phi_{g}}\right]$

$\operatorname{Im}\left(S_{V V} S_{H H}^{*}\right)=\operatorname{Im}\left[\sqrt{\sigma_{g t, V V}} \sqrt{\sigma_{g t, H H}} e^{i \Delta \phi_{g s}}\right]$

$+\operatorname{Im}\left[\sqrt{\sigma_{g, V V}} \sqrt{\sigma_{g, H H}} e^{i \Delta \phi_{g}}\right]$ 


\section{Dielectric Bevahior of Constituents}

- Vegetation - Ulaby and El-Rayes (1987)

$$
\begin{aligned}
\epsilon_{\mathrm{mg}}=A & +B\left(4.9+\frac{\epsilon_{s}-\epsilon_{\infty}}{1+j \frac{f(\mathrm{~Hz})}{f_{o}}}-\frac{22.74}{f(\mathrm{GHz})}\right) \\
& +C\left(2.9+\frac{55}{1+\sqrt{j \frac{f(\mathrm{GHz})}{0.18}}}\right)
\end{aligned}
$$

- Soil - Hallikainen et al. (1985)

$$
\begin{aligned}
\epsilon_{\mathrm{mv}} & =\epsilon_{s}^{\prime}-j \epsilon_{s}^{\prime \prime} \\
\epsilon & =\left(a_{0}+a_{1} S+a_{2} C\right) \\
& +\left(b_{0}+b_{1} S+b_{2} C\right) m_{v} \\
& +\left(c_{0}+c_{1} S+c_{2} C\right) m_{v}^{2} \\
\epsilon & =\epsilon_{s}^{\prime} \text { or } \epsilon_{s}^{\prime \prime}
\end{aligned}
$$




\section{Canopy Backscatter, $\theta=50^{\circ}$, HH Pol.}

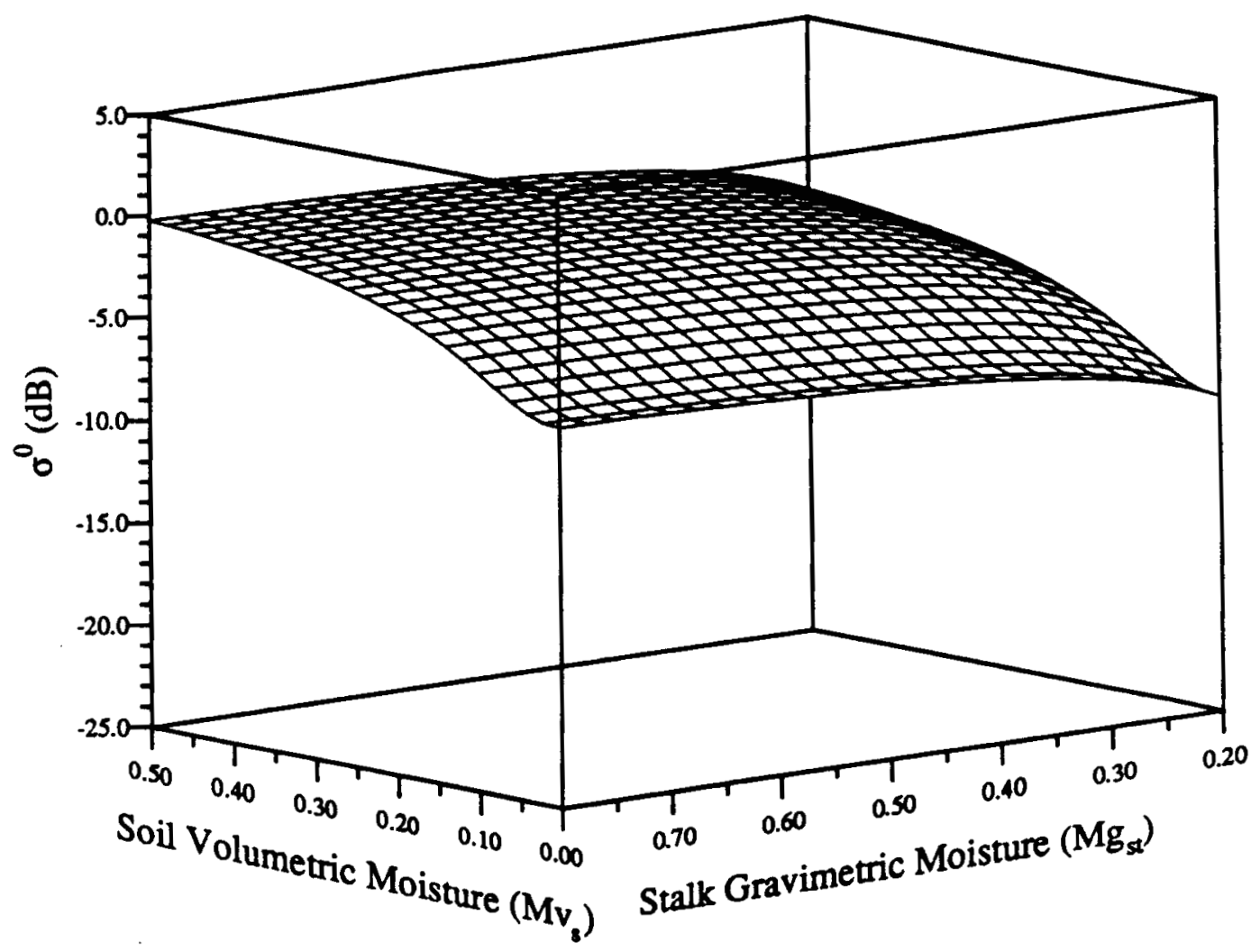

Canopy Backscatter, $\theta=50^{\circ}$, VV Pol.

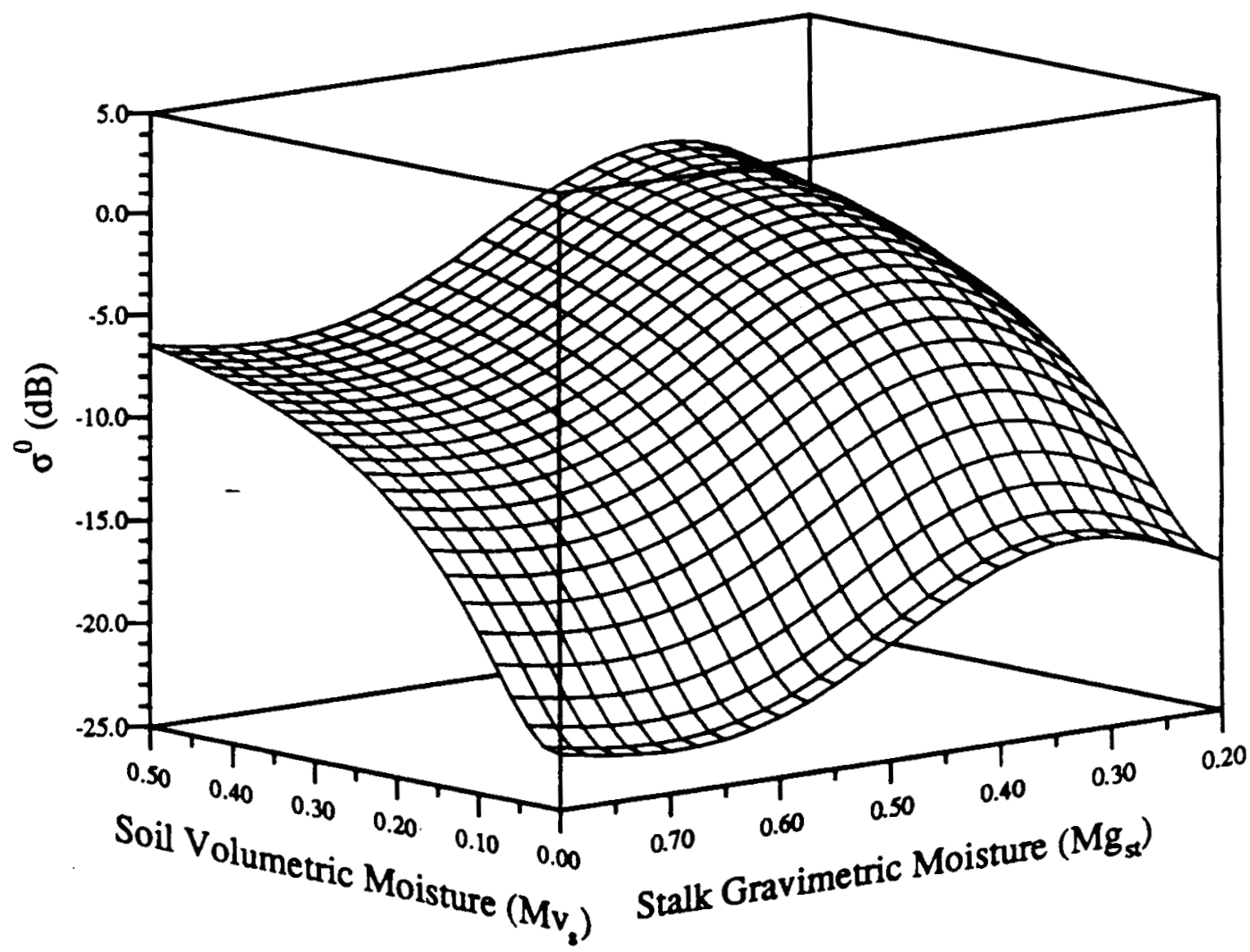


Polarization Phase Difference, $\theta=50^{\circ}$

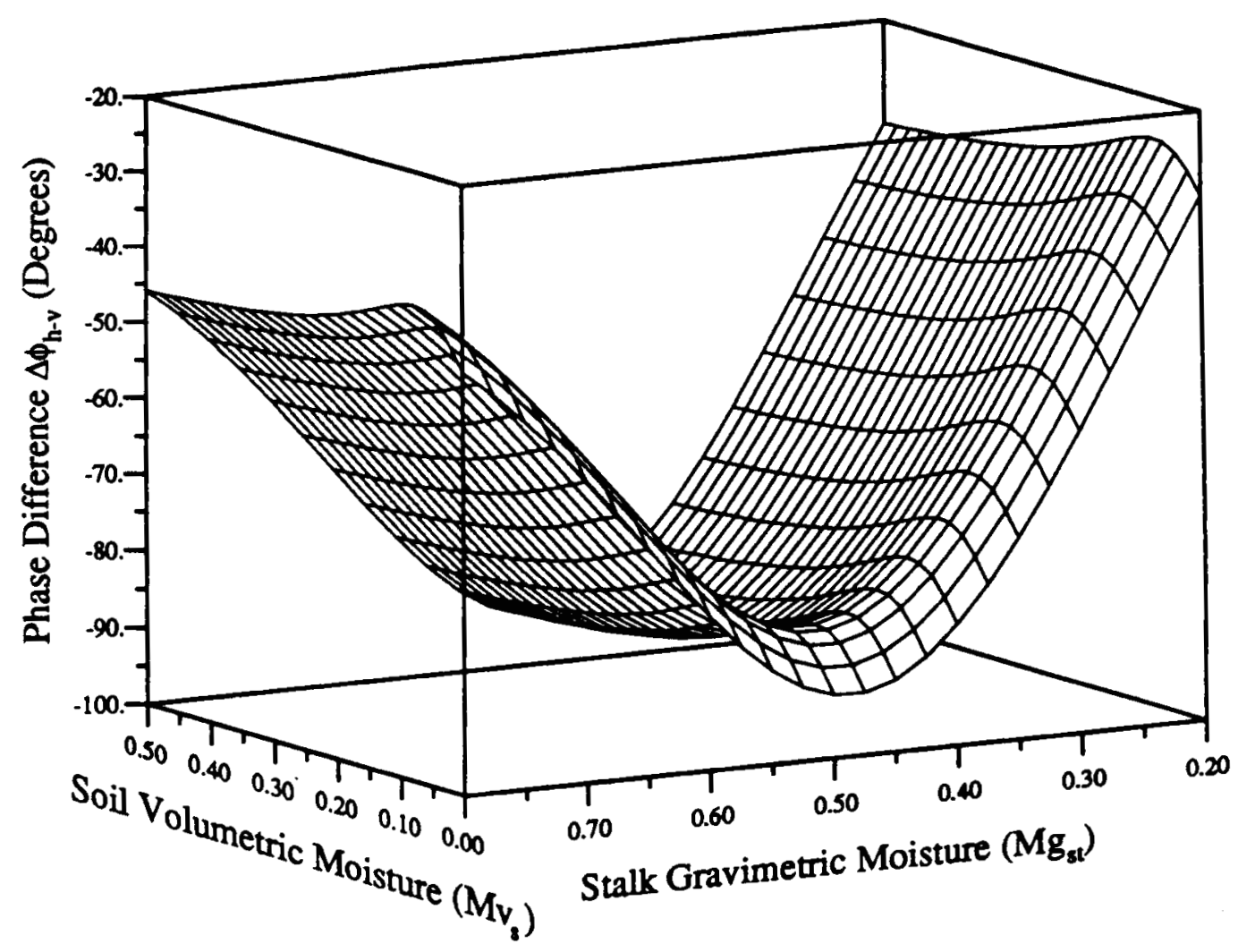




\section{Polarization Phase Difference, $\theta=30^{\circ}$}

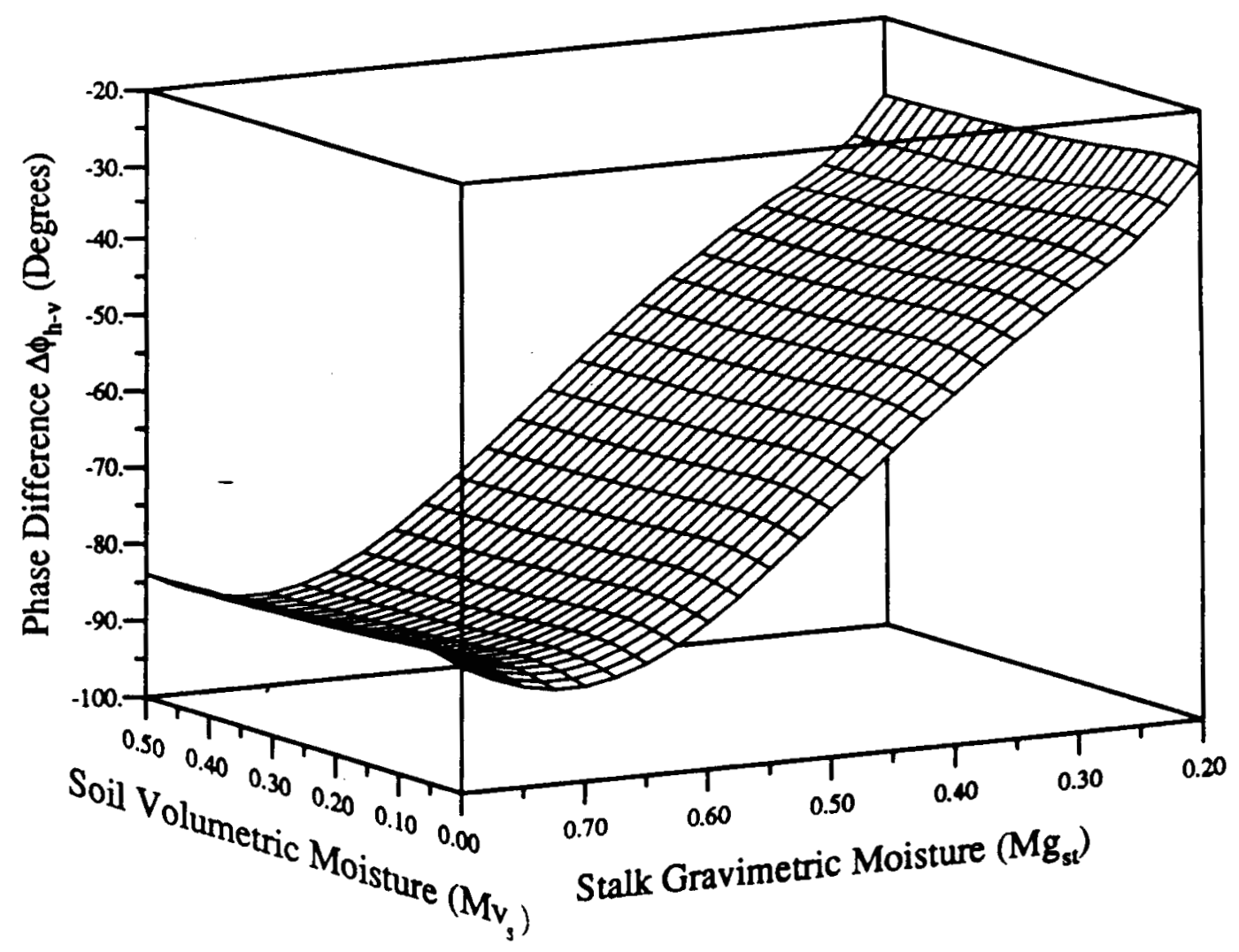




\section{Canopy Backscatter, $\theta=30^{\circ}$, HH Pol.}

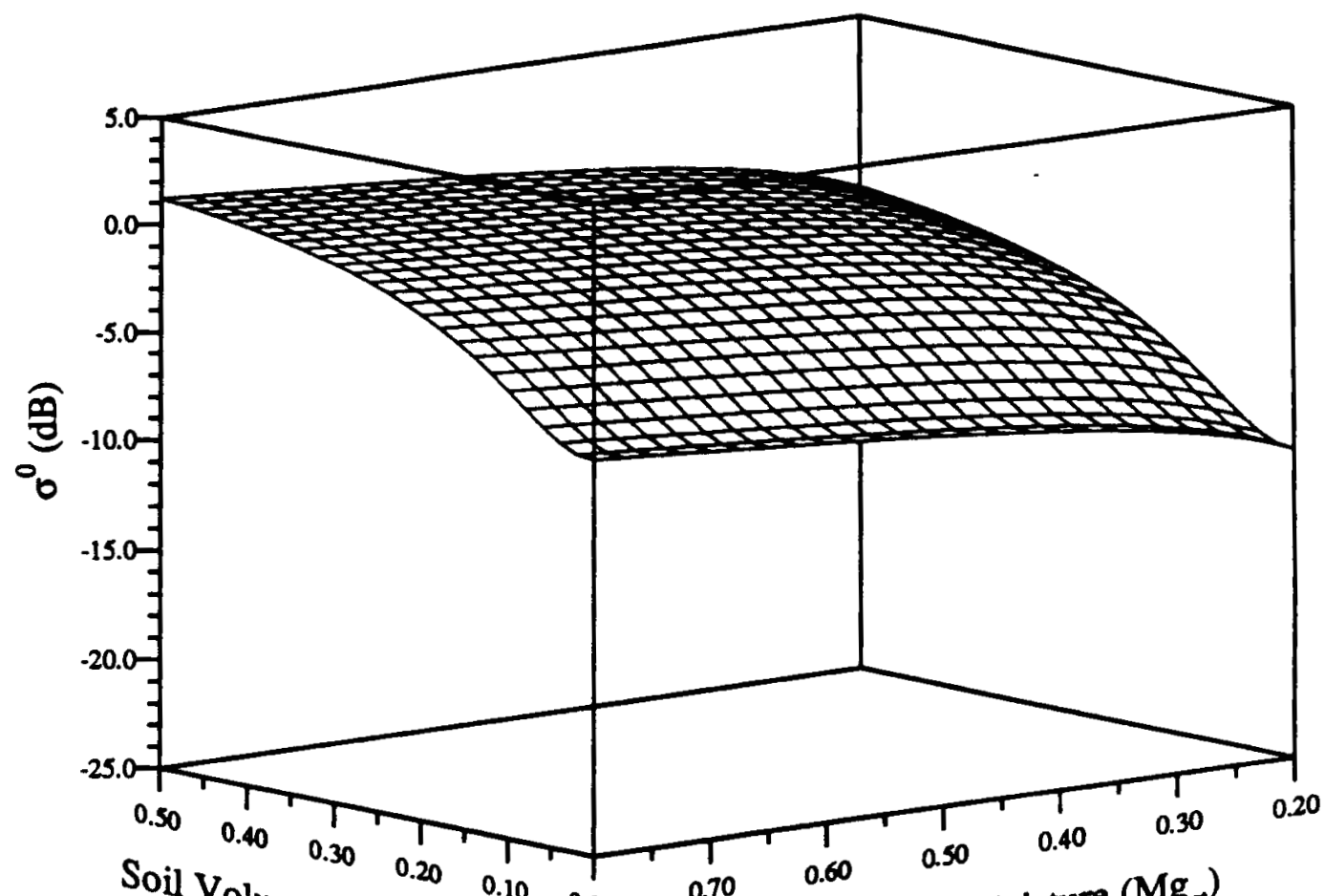

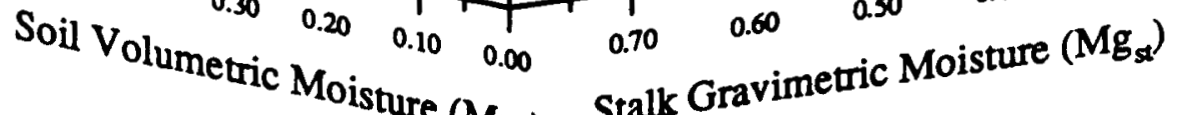

Moisture $\left(\mathrm{Mv}_{\mathrm{s}}\right)$ stalk Gravimetric Moist
Canopy Backscatter, $\theta=30^{\circ}$, VV Pol.

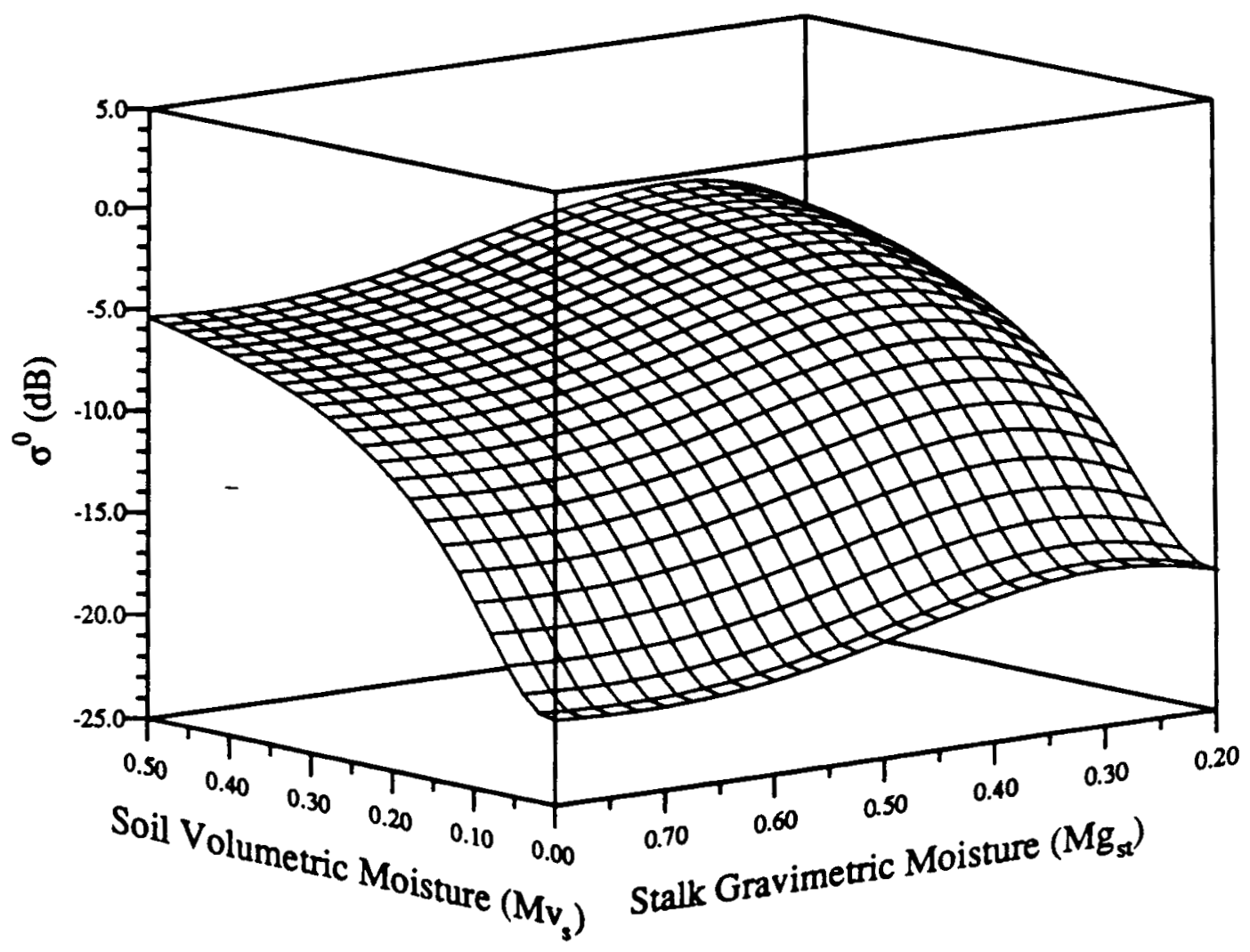


Moisture Parameters, $\theta=30$ Degrees
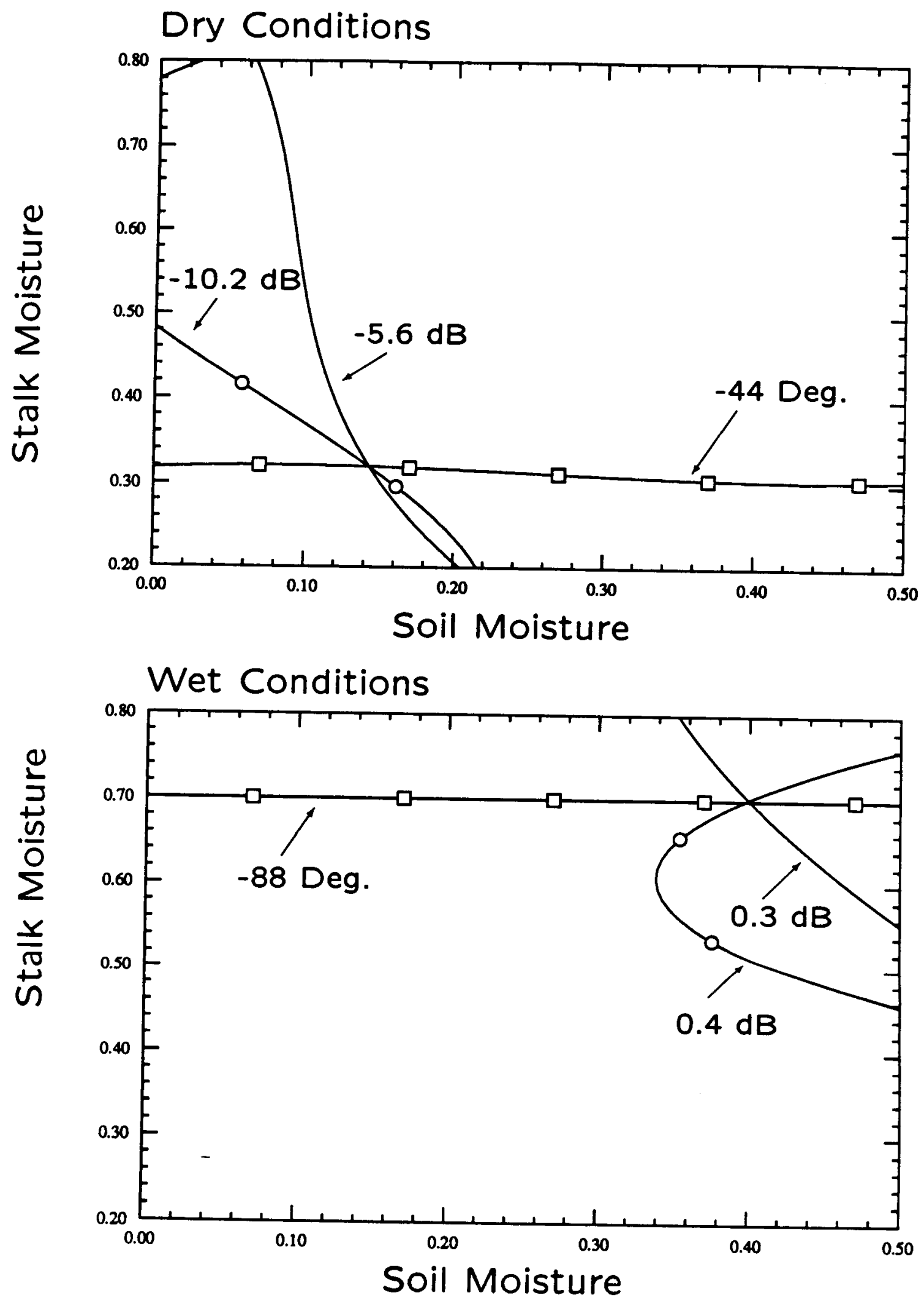

$\bar{\bigcirc} \sigma_{H H}$
$\sigma_{V V V} V$
$\Delta \phi_{H H-V V}$ 

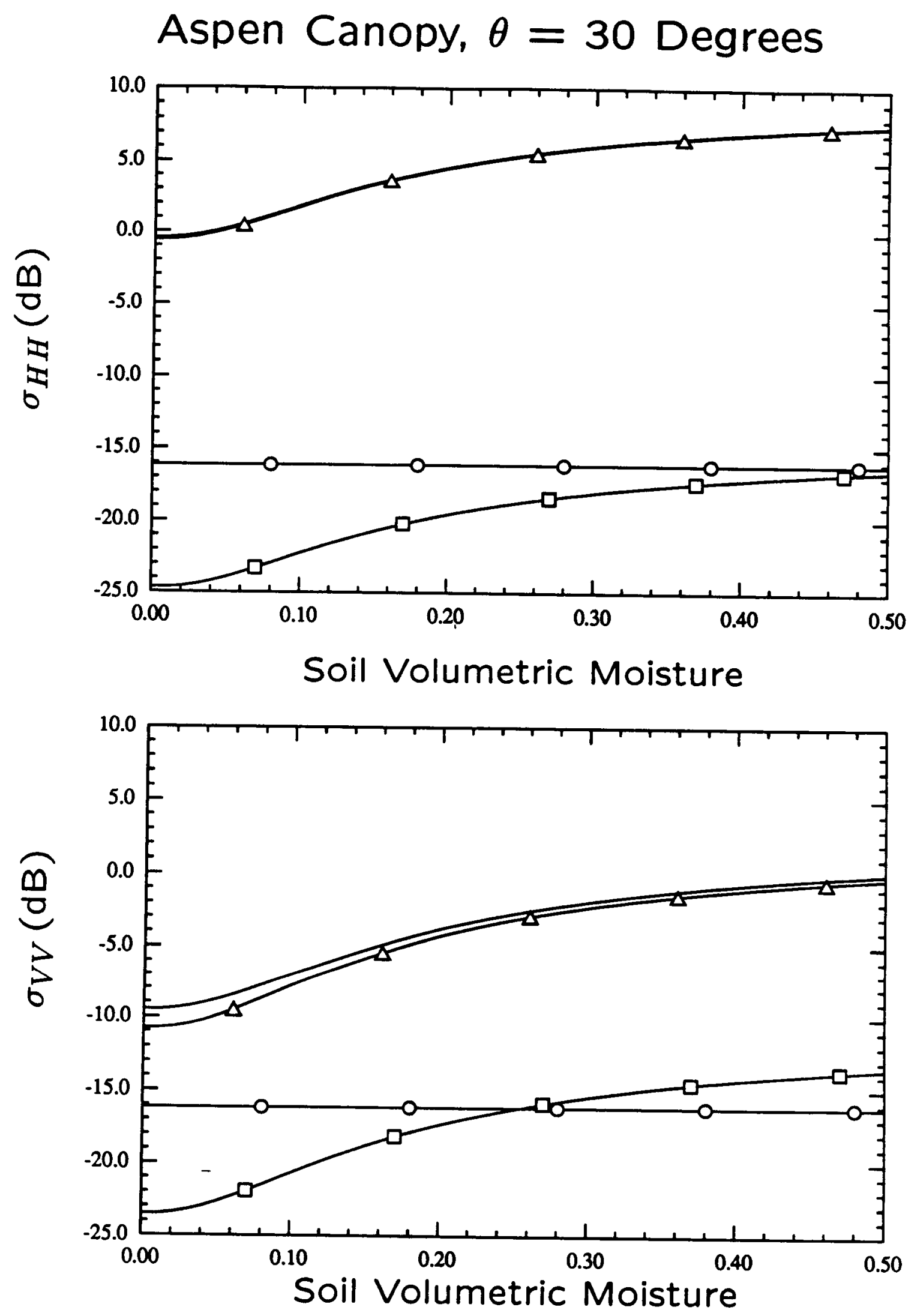

- Total Backscatter

ODirect Crown

$\square$ Direct Ground $\triangle$ Ground-Trunk 


\section{CALCULATED PHASE DIFFERENCE}

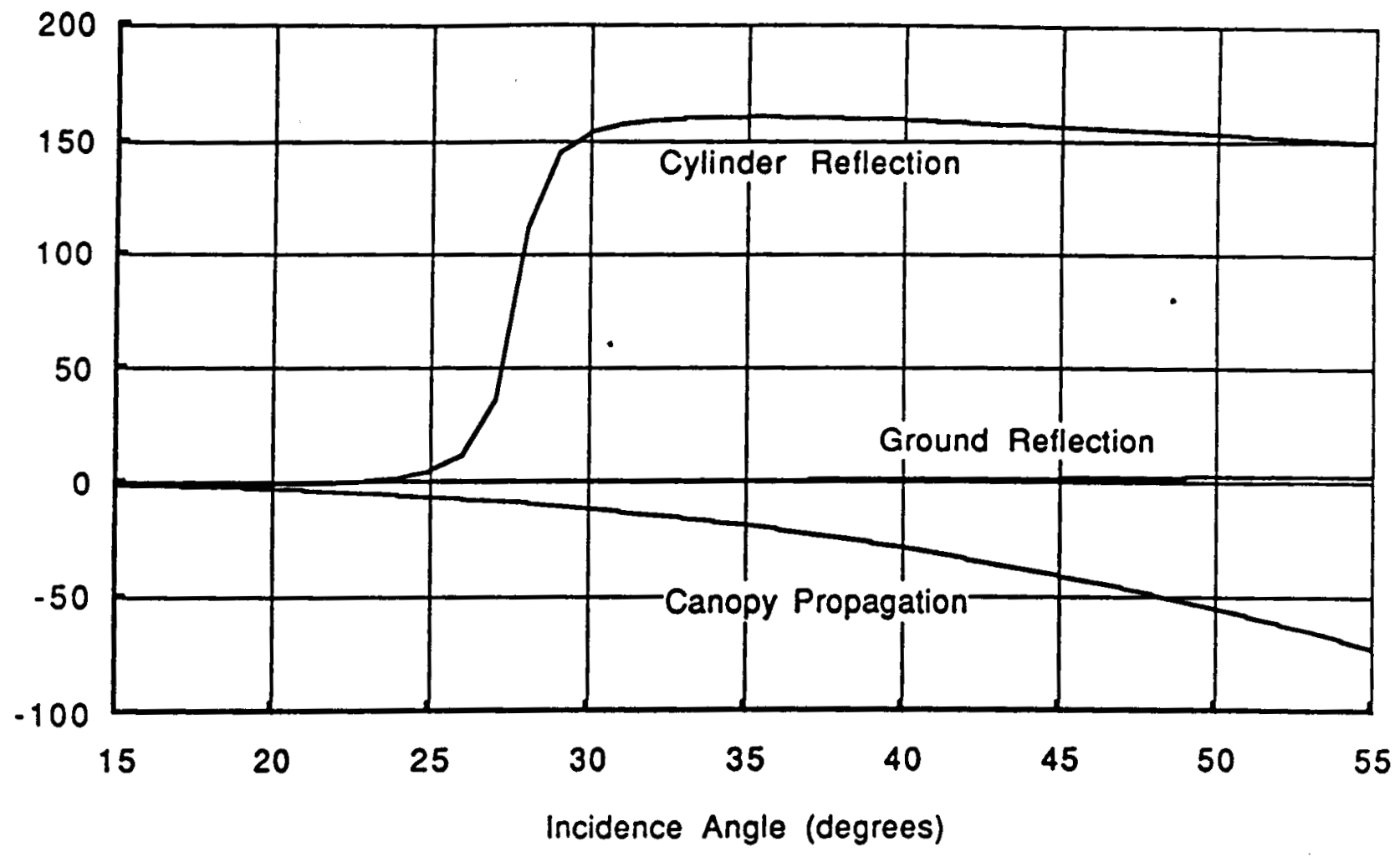

CALCULATED AND MEASURED PHASE DIfFERENCE

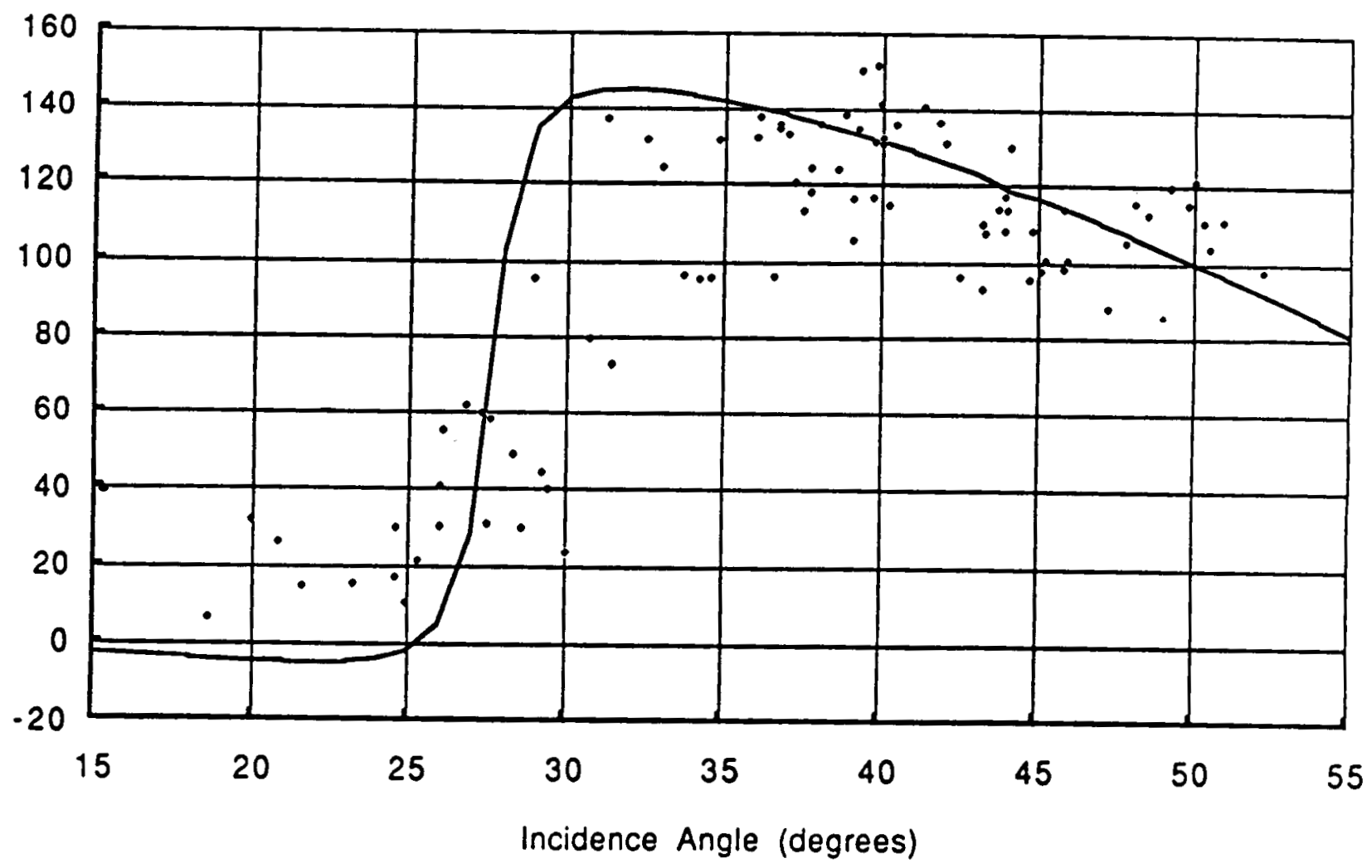


Backscatter Contours, $\theta=30$ Degrees
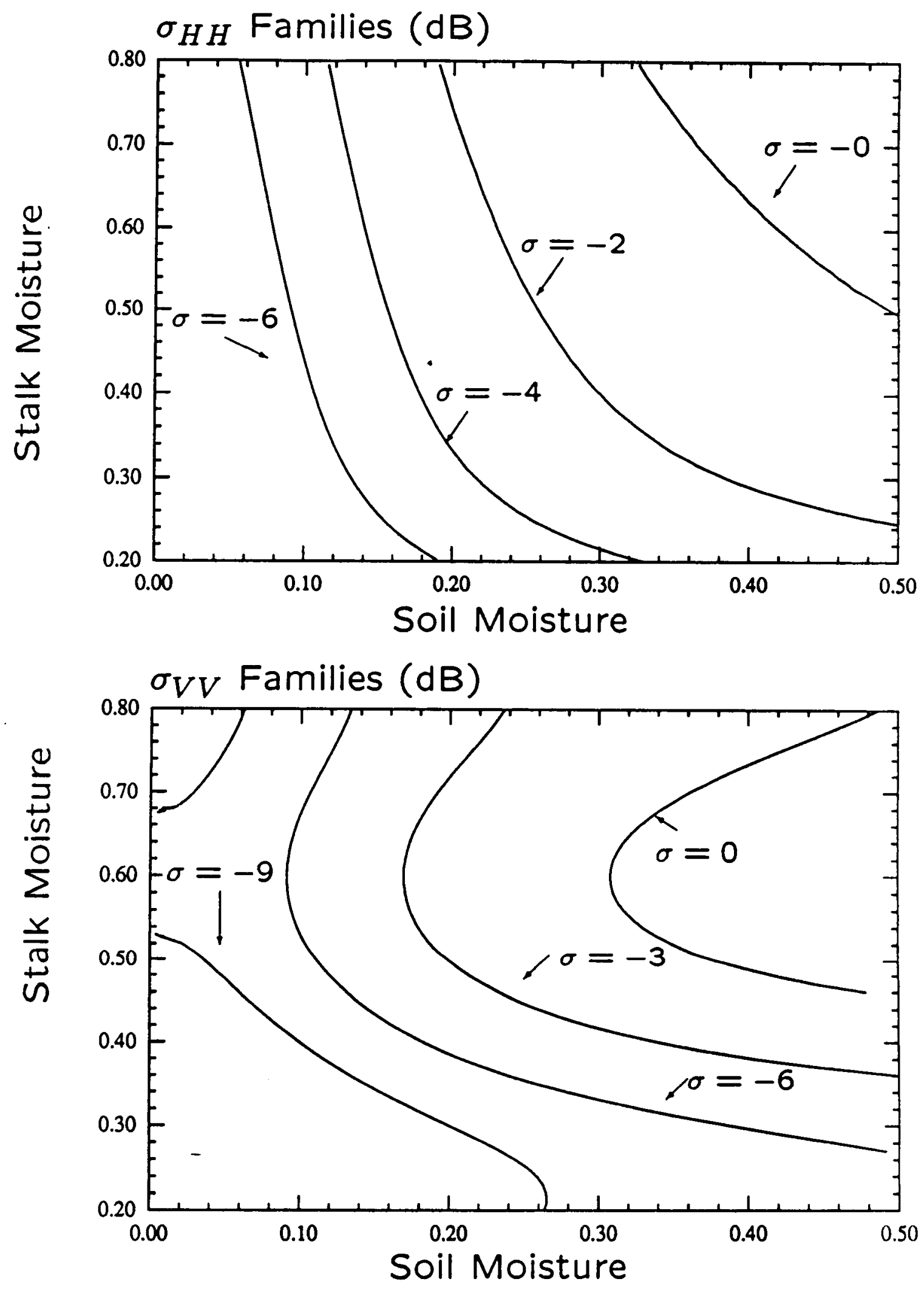
Phase Difference Contours, $\theta=30$ Degrees

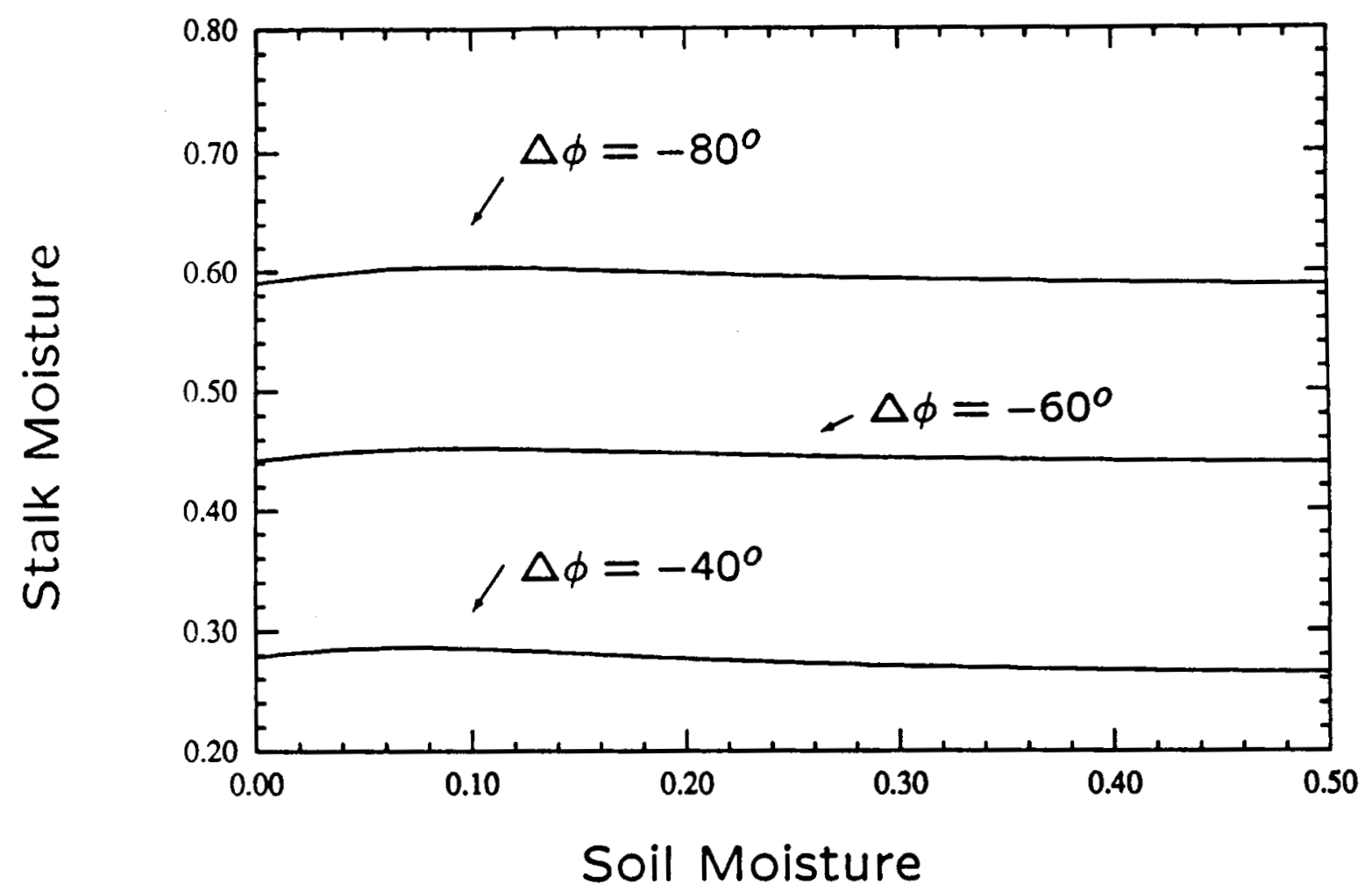




\section{Conclusions}

- Technique applies to canopies with large enough trunk/stalk biomass.

- Steep incidence angles are most effective.

- Polarization phase difference determines the trunk/stalk moisture.

- Polarization magnitudes determine soil moisture.

- $\mathrm{V}$ polarization magnitude is more effective than $\mathrm{HH}$. 